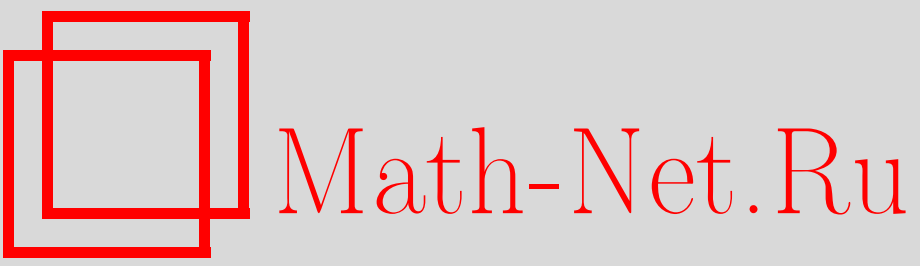

Н. Н. Ганиходжаев, Д. В. Занин, Об одном необходимом условии эргодичности квадратичных операторов, определенных на двумерном симплексе, УМH, 2004, том 59, выпуск 3, 161-162

DOI: https://doi.org/10.4213/rm744

Использование Общероссийского математического портала Math-Net.Ru подразумевает, что вы прочитали и согласны с пользовательским соглашением

http: //www . mathnet.ru/rus/agreement

Параметры загрузки:

IP : 18.209 .158 .208

26 апреля 2023 г., 17:23:28 


\title{
ОБ ОДНОМ НЕОБХОДИМОМ УСЛОВИИ ЭРГОДИЧНОСТИ КВАДРАТИЧНЫХ ОПЕРАТОРОВ, ОПРЕДЕЛЕННЫХ НА ДВУМЕРНОМ СИМПЛЕКСЕ
}

\author{
Н. Н. ГАНИХОДЖАЕВ, Д. В. ЗАНИН
}

1. Пусть $S^{m-1}=\left\{x=\left(x_{1}, x_{2}, \ldots, x_{m}\right) \in \mathbb{R}^{m}: x_{i} \geqslant 0, \sum_{i=1}^{m} x_{i}=1\right\}-(m-1)$-мерньй симплекс.

Отображение $V$ симплекса $S^{m-1}$ в себя назьвается квадратичным стохастическим оператором (к.с.о.), если для любого $x \in S^{m-1}$ и для всех $k=1, \ldots, m(V x)_{k}=\sum_{i, j=1}^{m} p_{i j, k} x_{i} x_{j}$, где $p_{i j, k} \geqslant 0, \sum_{k=1}^{m} p_{i j, k}=1$ и $p_{i j, k}=p_{j i, k}$ для всех $i, j, k$. Уламом на основе численных расчетов была сформулирована гипотеза [1], что для любого к.с.о. $V$ имеет место эргодическая теорема, т.е. для любого $x \in S^{m-1}$ существует предел $\lim _{n \rightarrow \infty} \frac{1}{n} \sum_{k=0}^{n-1} V^{k} x$. В 1977 году Захаревич [2] доказал, что это гипотеза в общем случае неверна.

K.c.o. $V$, определенный на $S^{m-1}$, назьвается волштерровским, если $p_{i j, k}=0$, как только $k$ отлично от $i$ и $j$, т.е. $k \notin\{i, j\}$.

В данной работе для класса волтерровских к.с.о., определенных на $S^{2}$, устанавливается необходимое условие того, чтобы имела место эргодическая теорема.

2. Любой вольтерровский к.с.о., определенный на $S^{2}=\{(x, y, z): x, y, z \geqslant 0, x+y+z=1\}$, можно представить следующим образом:

$$
V: x^{\prime}=x(1+a y-b z), \quad y^{\prime}=y(1-a x+c z), \quad z^{\prime}=z(1+b x-c y),
$$
где $a, b, c \in[-1,1]$.

ТЕОРема. Если для вольтерровского квадратичного стохастического оператора (*) все параметры $a, b, c$ имеют один и тот же знак и все они отличны от нуля, то для него не выполняется эргодическая теорема.

Точки $M_{0}=\left(\frac{c}{a+b+c}, \frac{b}{a+b+c}, \frac{a}{a+b+c}\right), M_{1}=(1,0,0), M_{2}=(0,1,0), M_{3}=$ $(0,0,1)$ являются неподвижными точками к.с.о. $V$.

Лемма 1. Пусть $M \in \operatorname{Int} S^{2}$ и $M \neq M_{0}$. Тогда предельное множество траектории точки $M$ бесконечно и лежит на граниче симплекса $S^{2}$.

ДоказАтЕльСтво. Обозначим $f(M)=x^{c} y^{b} z^{a}$, где $M=(x, y, z)$. Тогда $\frac{f(V M)}{f(M)}=$ $(1+a y-b z)^{c}(1-a x+c z)^{b}(1+b x-c y)^{a} \leqslant\left(1+\frac{1}{a+b+c}[c(a y-b z)+b(-a x+c z)+\right.$ $a(b x-c y)])^{a+b+c}=1$, так что $f\left(V^{k} M\right)$ убьвает с ростом $k$. Нетрудно показать, что $f\left(V^{k} M\right)$ сходится к нулю, т.е. пределнне множество лежит на $\partial S^{2}$. Так как вольтерровские операторы оставляют неподвижными вершины и инвариантными ребра $S^{2}$, то предельное множество не может быть конечньм.

Введем следующие обозначения: $G_{1}=\left\{(x, y, z) \in S^{2}: x \succ y \succ z\right\}, G_{2}=\{x \succ z \succ y\}$, $G_{3}=\{z \succ x \succ y\}, G_{4}=\{z \succ y \succ x\}, G_{5}=\{y \succ z \succ x\}, G_{6}=\{y \succ x \succ z\}$, где " $x \succ y$ " означает, что $b x \geqslant c y$, " $x \succ z$ " означает, что $a x \geqslant c z$ и " $y \succ z$ " означает, что $b y \geqslant c z$.

ЛЕмма 2. Для любой точки $M \in G_{1}$, отличной от неподвижной, маршрут ее движения по $S^{2}$ задается диаграммой $G_{1} \rightarrow G_{2} \rightarrow G_{3} \rightarrow G_{4} \rightarrow G_{5} \rightarrow G_{5} \rightarrow G_{6} \rightarrow G_{1}$.

ДокАЗАТЕльство. Для $M \in G_{1}$ координаты точки $V M=\left(x^{\prime}, y^{\prime}, z^{\prime}\right)$ удовлетворяют неравенствам $x^{\prime} \geqslant x, y^{\prime} \leqslant y, z^{\prime} \geqslant z$, так что $V M \in G_{1} \cup G_{2}$. Тогда после некоторого времени траектория попадает в $G_{2}$. Аналогично рассматриваются остальные случаи.

Выберем теперь такую окрестность $U_{0}$ точки $M_{0}$, что $U_{0} \subset \operatorname{Int} S^{2}$ и $U_{1}=\left(G_{1} \cup G_{2}\right) \backslash U_{0}$,

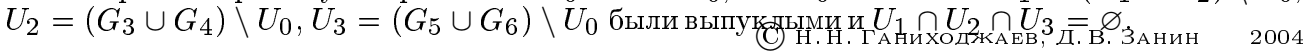


ЛЕмма 3. Пусть $M \notin U, V^{k} M \in U$ для всех $k=1,2, \ldots, n u V^{n+1} M \notin U$, где $U$ - одна из областей $U_{i}, M \in \operatorname{Int} S^{2}$ и $M \neq M_{0}$. Тогда $n \geqslant A \ln \frac{B}{f(V M)}$, где $A, B-$ абсолютнье Константь.

ДокаЗАтельство. Пусть, например, $U=U_{1}$. Тогда $M \notin U_{1}$, откуда $M \in G_{6}$, т.е. $y \succ x \succ$ $z$. Так как $V M \in U_{1}$, то $x^{\prime} \succ y^{\prime} \succ z^{\prime}$, откуда $x^{\prime} \geqslant \frac{\min (a, b, c)}{a+b+c}=\alpha$.

a) $\frac{y^{\prime}}{x^{\prime}} \geqslant \min _{M \in G_{6}} \frac{y^{\prime}}{x^{\prime}}=\beta \geqslant 0$. Очевидно, $\beta \neq 0$, так как если $\beta=0$, то сушествует $M \in G_{6}$, для которой $\frac{y^{\prime}}{x^{\prime}}=0$, т.е. $y(1-a x+c z)=0$. Если $y=0$, то $y \succ x \succ z$, откуда $x=z=0$, что невозможно. Если $1-a x+c z=0$, то $z=0, a x=1$, т.е. $a=x=1$, откуда $y=0$, но $y \succ x$, что невозможно.

б) Положим $V^{k} M=\left(x_{k}, y_{k}, z_{k}\right)$. Так как $V^{n+1} M \notin U_{1}$, то $z_{n+1} \succ x_{n+1} \succ y_{n+1}$, откуда $z_{n+1} \geqslant \frac{\min (a, b, c)}{a+b+c}=\alpha$.

в) $\frac{z_{n+1}}{z_{1}}=\prod_{k=1}^{n} \frac{z_{k+1}}{z_{k}}=\prod_{k=1}^{n}\left(1+b x_{k}-c y_{k}\right) \leqslant 2^{n}$. Тогда $2^{n} \geqslant \frac{z_{n+1}}{z_{1}} \geqslant \frac{\alpha}{z_{1}}=$ $\left(\frac{\alpha^{a}}{z_{1}^{a}}\right)^{1 / a}=\left(\frac{\alpha^{a} x_{1}^{c} y_{1}^{b}}{f(V M)}\right)^{1 / a} \geqslant\left(\frac{\alpha^{a} \beta^{b} x_{1}^{b+c}}{f(V M)}\right)^{1 / a}$, откуда $n>A \ln \frac{B}{f(V M)}$.

Лемма 4. Пусть $U-$ одна из областей $U_{i}, M \in \operatorname{Int} S^{2}, M \neq M_{0}$. Пусть $\left\{n_{i}, m_{i}\right\}_{i=1}^{\infty}$ - последовательность натуральных чисел таких, что $V^{n_{i}} M \in U, V^{n_{i}+k} M \in U$ для $k=1, \ldots, m_{i}$ u $V^{n_{i}+m_{i}+1} \notin U$. Тогда существует с такое, ито $m_{i}>c n_{i}$.

ДокАЗАТЕльство. Пусть $\rho=\max _{S^{2} \backslash U_{0}}(1+a y-b z)^{c}(1-a x+c z)^{b}(1+b x-c y)^{a}<1$. Применим лемму 3 с учетом того, что $\frac{1}{f(V M)}>C\left(M_{0}\right) \rho^{n_{i}}$, где $n=m_{i}$, а $C$ зависит от $M_{0}$.

ДОКАЗАТЕЛЬСтво тЕОРЕМЫ. Предположим, что для любой точки $M \in \operatorname{Int} S^{2}, M \neq M_{0}$, существует предел $\lim _{n \rightarrow \infty} \frac{1}{n} \sum_{k=0}^{n-1} V^{k} M=M^{*}$. Пусть $M^{*} \notin U_{1},\left\{n_{i}, m_{i}\right\}$ - такие, как в лемме 4. Пусть $\delta=\operatorname{dist}\left(M^{*}, U_{1}\right)$ и $\operatorname{dist}\left(\frac{1}{n} \sum_{k=0}^{n} V^{k} M, M^{*}\right)<\frac{\delta}{e}$ при достаточно больших $n$. Так как $\lambda_{i}=\frac{m_{i}}{n_{i}}>c$, то для $M^{\prime}=\frac{1}{1+\lambda_{i}}\left(\frac{1}{n_{i}} \sum_{k=0}^{n_{i}} V^{k} M\right)+\frac{\lambda_{i}}{1+\lambda_{i}}\left(\frac{1}{m_{i}} \sum_{k=n_{i}+1}^{n_{i}+m_{i}} V^{k} M\right)$

$$
\begin{aligned}
& \operatorname{dist}\left(M^{\prime}, M^{*}\right) \geqslant \operatorname{dist}\left(\frac{1}{n_{i}} \sum_{k=0}^{n_{i}} V^{k} M, M^{\prime}\right)-\frac{\delta}{e} \\
& =\frac{\lambda_{i}}{1+\lambda_{i}} \operatorname{dist}\left(\frac{1}{n_{i}} \sum_{k=1}^{n_{i}} V^{k} M, \frac{1}{m_{i}} \sum_{k=n_{i}+1}^{n_{i}+m_{i}} V^{k} M\right)-\frac{\delta}{e} \geqslant \frac{c}{1+c} \operatorname{dist}\left(\frac{1}{n_{i}} \sum_{k=1}^{n_{i}} V^{k} M, U_{1}\right)-\frac{\delta}{e} \\
& \geqslant \frac{c}{1+c}\left(\operatorname{dist}\left(M^{*}, U_{1}\right)-\frac{\delta}{e}\right)-\frac{\delta}{e}=\left(\frac{c}{1+c}-\frac{1}{e} \frac{2+c}{1+c}\right) \delta>\frac{\delta}{e}
\end{aligned}
$$

для достаточно больших $e$, что приводит к противоречию. Теорема доказана.

\section{СПИСОК ЛИТЕРАТУРЫ}

[1] С. Улам. Нерешенные математические задачи. М.: Наука, 1964. [2] М. И. Захаревич // УМH. 1978. Т. 33. №6. C. 207-208.

Принято редколлегией 01.04 .2004 